\title{
COMUNICAÇÃO SOCIAL E SAÚDE: CONSCIENTIZAÇÃO E INCENTIVO A IMUNIZAÇÃO
}

\section{ARTIGO DE REVISÃO}

SANTOS, Gustavo Gonçalves dos ${ }^{1}$

SANTOS, Vitória Gonçalves dos ${ }^{2}$

SANTOS, Gustavo Gonçalves dos. SANTOS, Vitória Gonçalves dos. Comunicação social e saúde: Conscientização e incentivo a imunização. Revista Científica Multidisciplinar Núcleo do Conhecimento. Ano 04, Ed. 10, Vol. 06, pp. 64-78. Outubro de 2019. ISSN: 2448-0959, Link de acesso: https://www.nucleodoconhecimento.com.br/saude/incentivo-a-imunizacao

\section{RESUMO}

Objetivo: Descrever sobre o poder Comunicação Social na Enfermagem em relação ao incentivo e conscientização em relação às campanhas de vacinação. Método: Trata-se de uma revisão bibliográfica, realizada no período de Fevereiro à Julho de 2019. Foram utilizados 10 estudos como referencial teórico relativo à Saúde e Comunicação Social com ênfase na Publicidade e Propaganda por meio de fontes como publicações do Ministério da Saúde, Organização Mundial da Saúde e artigos de periódicos científicos publicados nos últimos dez anos, disponibilizados na

\footnotetext{
${ }^{1}$ Enfermeiro e Docente. Graduado em Enfermagem pela Escola de Ciências da Saúde da Universidade Anhembi Morumbi. Pós-graduando Enfermagem Obstétrica e Ginecológica pela Faculdade Israelita de Ciências da Saúde do Hospital Albert Einstein - Instituo Israelita de Ensino e Pesquisa. Pós-graduando Enfermagem em Saúde Pública pela Escola Paulista de Enfermagem da Universidade Federal de São Paulo.

${ }^{2}$ Graduanda em Comunicação Social - Publicidade e Propaganda pela Escola de Comunicação da Universidade Anhembi Morumbi.
} 
Biblioteca Virtual: SciELO, LILACS, BDEFN e Google Acadêmico, utilizando os descritores: Calendário Vacinal, Imunização, Comunicação Social. Resultados: A Comunicação Social em saúde oferece condições significantes e relevantes para as boas práticas de promoção à saúde, favorecendo o estímulo do autocuidado e o cuidado dos usuários do serviço de saúde. Conclusões: Mobilizar profissionais da saúde e os usuários dos serviços de saúde no que se diz respeito à conscientização e incentivo da imunização.

Descritores: calendário vacinal, imunização, comunicação social.

\section{INTRODUÇÃO}

Imunização é uma ação de caráter preventiva, reconhecida pela redução nas taxas de mortalidade. No Brasil, houve a criação do Programa Nacional de Imunizações, instituído pela Lei oㅜ 6.59/75, lei essa que regulamenta as ações de vigilância, imunização e notificação compulsória de doenças. As vacinas são praticadas de maneira sistemática e ofertadas de forma gratuita por entidades públicas, mas também por entidades privadas. ${ }^{(1)}$

O Art. 27 da Lei ํㅜ 6.59/75 regulamenta que as vacinas são obrigatórias em todo o território nacional, as vacinas como tal que são definidas pelo Programa Nacional de Imunizações. O decreto ainda dispõe no Art. 29 que é dever de todo cidadão submeter-se a imunização, juntamente com os menores dos quais tenha guarda e/ou responsabilidade. ${ }^{(1)}$

A imunização consiste em uma das ações preventivas de Saúde Pública mais segura, econômica e efetiva capaz de prevenir mortalidade e oferecer qualidade de vida, em especial às populações de maior vulnerabilidade. ${ }^{(1,2)}$

Tratar com descuido o Calendário Vacinal pode causar agravos à saúde, agravos estes que podem se reverter em maiores problemas de Saúde Pública. Um apoio no que se diz respeito à atuação de primeira linha em relação à imunização, é a Atenção Primária à Saúde que é considerada um importante meio de atuação no que se refere 
à maneira segura e efetiva o controle e/ou erradicação de doenças agravantes à saúde. (3)

À medida que aumenta o número de vacinas e o seu uso pelos Programas de Saúde Pública, cresce também a quantidade de pessoas e grupos que declaram preocupações com a segurança e a necessidade da aplicação das vacinas. Pais e/ou responsáveis, cuidadores, pacientes e os próprios profissionais da saúde que atuam diretamente nesta perspectiva. No Brasil, o Programa Nacional de Imunizações conta com a credibilidade e respeitabilidade da população, da comunidade científica e também da comunidade da Comunicação Social. ${ }^{(4)}$

A vacinação é considerada um custo-efetivo e segura, favorece proteção individual e coletiva, estando diretamente ligada a promoção de saúde, proteção e manutenção da saúde. O Ministério de Saúde tem oferecido ações, como o Dia D, com intuito de imunizar o maior número de indivíduos, principalmente crianças e idosos que se enquadram como indivíduos mais suscetíveis a doenças. ${ }^{\left({ }^{5}\right)}$

Diante do que foi exposto surgi a seguinte indagação: Qual a finalidade da Comunicação Social em Saúde no que se diz respeito à conscientização e incentivo para imunização? Respondendo tal questionamento este estudo tem como objetivo descrever sobre a Comunicação Social em Saúde, em relação ao incentivo e conscientização em relação vacinação e descrever a relevância da Comunicação Social na Enfermagem no que se diz respeito à imunização da população.

\section{MÉTODO}

Trata-se de uma revisão integrativa, método este utilizado desde 1980 permitindo buscar, avaliar e elaborar uma síntese de evidências utilizando variados estudos sobre o tema estudado nesta presente revisão, possibilitando então o levantamento de lacunas que precisam ser preenchidas com publicação de novos estudos. ${ }^{(13)}$

O percurso metodológico seguiu as seguintes etapas: Identificação do tema, elaboração da pergunta norteadora; definição dos critérios para inclusão e exclusão 
de estudos; busca de artigos científicos na literatura; avaliação dos estudos incluídos na presente revisão e interpretação dos resultados. No que se diz respeito à questão norteadora, foi indagado: Qual a finalidade da Comunicação Social em Saúde no que se diz respeito à conscientização e incentivo para imunização?

Realizou-se o presente estudo no período de Fevereiro à Julho de 2018, buscando artigos científicos com metodologia de Revisões sistemáticas da literatura, estudos exploratório-descritivos, estudos qualitativos e estudos quantitativos nas bases de dados da Literatura Latino-Americana e do Caribe em Ciências da Saúde (LILACS), Scientific Eletronic Library Online (SciELO), Base de dados de Enfermagem (BDENF) e Google Acadêmico, utilizando os seguintes descritores: Calendário Vacinal, Imunização, Comunicação Social, Publicidade e Propaganda, Educação em Enfermagem. Após a busca em bases de dados, foi selecionado um total de 20 (vinte) artigos. Posteriormente foi realizada leitura na íntegra dos artigos selecionados, sendo incluídos na presente revisão 10 (dez) artigos, que abordavam com relevância a temática deste estudo e se enquadrava dentro dos critérios de inclusão estabelecidos.

Para os critérios de inclusão dos estudos foram estabelecidos os seguintes: período das publicações entre os anos de 2010 a 2018; publicações no idioma Português e Espanhol e artigos indexados na íntegra nas bases de dados selecionadas.

E para os critérios de exclusão foram adotados os seguintes: artigos repetidos em mais de uma base de dados, artigos que não foram publicados nos últimos dez anos e artigos no idioma Inglês.

Foi elaborado um quadro, contendo referência e ano, autores, título do artigo e objetivos. Os dados incluídos neste estudo foram analisados de forma descritiva, a partir de trechos extraídos dos estudos incluídos nesta pesquisa. Utilizando a análise temática de Conteúdo de Bardin, onde se encontram variados tipos de investigação, apoiados em diferentes técnicas metodológicas. A análise temática adotada neste estudo indica que a mesma já era utilizada desde as primeiras tentativas da humanidade de interpretar os livros sagrados, tendo sido sistematizada como método apenas na década de 20, por Leavel. Ou seja, utilizando essa análise, o pesquisador 
busca compreender as características, estruturas ou modelos que estão por trás dos fragmentos da literatura tornados em consideração. ${ }^{(13,14)}$

Fluxograma do percurso metodológico, São Paulo - SP, Brasil. 2019.

Etapa 1: Identificação do tema e elaboração da pergunta norteadora
Etapa 2: Estabelecimento de critérios para inclusão e exclusão de estudos
Etapa 3: Busca na literatura, bases de dados LILACS, SciELO, BDENF e Google Acadêmico
Etapa 4: Avaliação dos estudos incluídos na revisão. Selecionados num total 20 estudos, sendo incluídos 10 que abordagem com relevância a temática
Etapa 5: Interpretação dos resultados por meio descritivo, a partir dos trechos que configuram evidência científica

Fonte: Dados do estudo, 2019.

\section{RESULTADOS E DISCUSSÃO}

Na presente revisão integrativa foram analisados 10 (dez) artigos, pois atenderam aos critérios de inclusão, conforme apresentado no Quadro 1. As fontes de publicação foram extraídas de diversos periódicos, os quais destacaram a área da Enfermagem, Comunicação em Saúde e Comunicação Social com ênfase na Publicidade e Propaganda.

Quadro 1 - Identificação dos estudos segundo referência, autores, título e objetivos. São Paulo - SP, Brasil. 2019.

\begin{tabular}{|l|l|l|l|}
\hline Referência & Autores & Título & Objetivos \\
\hline $\mathbf{1}$ & Barbieri CLA et & A (não) vacinação & Compreender como pais e \\
& al & infantil entre a cultura e & mães pertencentes às \\
& & a lei: os significados & camadas médias de São \\
& & atribuídos por casais de & Paulo significam as \\
\hline
\end{tabular}




\begin{tabular}{|c|c|c|c|}
\hline & & $\begin{array}{l}\text { camadas médias de } \\
\text { São Paulo, Brasil }\end{array}$ & $\begin{array}{l}\text { normatizações da } \\
\text { vacinação no país, a partir } \\
\text { de suas vivências de } \\
\text { vacinar, selecionar ou não } \\
\text { vacinar os filhos }\end{array}$ \\
\hline 2 & Silva FS et al & $\begin{array}{l}\text { Incompletude vacinal } \\
\text { infantil de vacinas } \\
\text { novas e antigas e } \\
\text { fatores associados: } \\
\text { coorte de nascimento } \\
\text { BRISA, São Luís, } \\
\text { Maranhão, Nordeste do } \\
\text { Brasil }\end{array}$ & $\begin{array}{l}\text { Analisar fatores } \\
\text { associados para a } \\
\text { incompletude de vacinas } \\
\text { de introdução recente. }\end{array}$ \\
\hline 3 & $\begin{array}{l}\text { Ramos CF et } \\
\text { al }\end{array}$ & $\begin{array}{l}\text { Cumprimento do } \\
\text { calendário de } \\
\text { vacinação de crianças } \\
\text { em uma unidade de } \\
\text { saúde da família }\end{array}$ & $\begin{array}{l}\text { Analisar o cumprimento do } \\
\text { calendário de vacinação } \\
\text { de crianças pelos usuários } \\
\text { da Unidade de Saúde da } \\
\text { Família Água bem como } \\
\text { possíveis razões para o } \\
\text { seu eventual não } \\
\text { cumprimento }\end{array}$ \\
\hline 4 & Mizuta $\mathrm{AH}$ et al & $\begin{array}{l}\text { Percepções acerca da } \\
\text { importância das vacinas } \\
\text { e da recusa vacinal } \\
\text { numa Escola de } \\
\text { Medicina }\end{array}$ & $\begin{array}{l}\text { Identificar a percepção da } \\
\text { importância das vacinas e } \\
\text { os riscos da recusa vacinal } \\
\text { entre alunos de Medicina } \\
\text { e médicos }\end{array}$ \\
\hline 5 & $\begin{array}{l}\text { Martins } \mathrm{KM} \text {, } \\
\text { Santos } \mathrm{WL} \text {, } \\
\text { Álvares } \mathrm{ACM}\end{array}$ & $\begin{array}{l}\text { A importância da } \\
\text { imunização: revisão } \\
\text { integrativa }\end{array}$ & $\begin{array}{l}\text { Proteger a gestante e o } \\
\text { recém-nascido, pois a } \\
\text { transmissão perinatal } \\
\text { representa uma das vias } \\
\text { mais convincentes de }\end{array}$ \\
\hline
\end{tabular}




\begin{tabular}{|c|c|c|c|}
\hline & & & $\begin{array}{l}\text { transmissão de doenças, } \\
\text { e a que mais } \\
\text { repentinamente leva a } \\
\text { sequelas e óbitos }\end{array}$ \\
\hline 6 & $\begin{array}{l}\text { Maia JÁ, } \\
\text { Menezes FA, } \\
\text { Santos PAM }\end{array}$ & $\begin{array}{l}\text { Percepção dos pais } \\
\text { sobre a importância de } \\
\text { acompanhamento do } \\
\text { crescimento e } \\
\text { desenvolvimento infantil }\end{array}$ & $\begin{array}{l}\text { Explicar a importância do } \\
\text { acompanhamento do } \\
\text { crescimento e do } \\
\text { desenvolvimento infantil } \\
\text { de crianças de um mês a } \\
\text { cinco anos }\end{array}$ \\
\hline 7 & $\begin{array}{l}\text { De Morais } \\
\text { Júnior SLA et. } \\
\text { al }\end{array}$ & $\begin{array}{l}\text { A evolução histórica do } \\
\text { calendário vacinal } \\
\text { brasileiro infantil. }\end{array}$ & $\begin{array}{l}\text { Apresentar a evolução do } \\
\text { calendário vacinal infantil } \\
\text { no Brasil }\end{array}$ \\
\hline 8 & $\begin{array}{l}\text { Coriolano- } \\
\text { Marinus MWL } \\
\text { et al }\end{array}$ & $\begin{array}{l}\text { Comunicação nas } \\
\text { práticas em saúde: } \\
\text { revisão integrativa da } \\
\text { literatura }\end{array}$ & 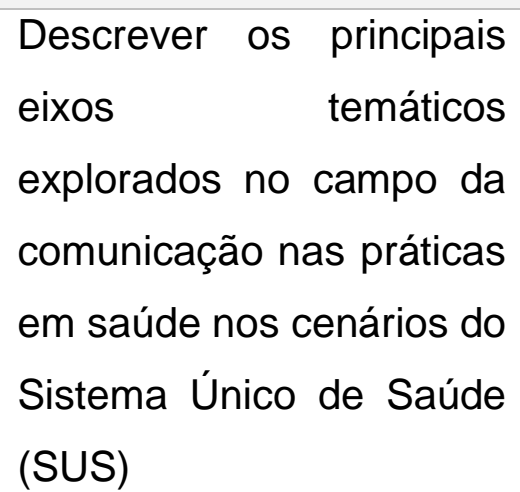 \\
\hline 9 & $\begin{array}{l}\text { Vasconcellos- } \\
\text { Silva PR et al }\end{array}$ & $\begin{array}{l}\text { A sociedade de risco } \\
\text { midiatizada, } \\
\text { movimento } \\
\text { antivacinação e o risco } \\
\text { do autismo }\end{array}$ & $\begin{array}{l}\text { Observar modificações } \\
\text { epidemiológicas de } \\
\text { doenças infecciosas entre } \\
\text { famílias de classe média } \\
\text { de países industrializados } \\
\text { por força de crenças } \\
\text { ligadas aos riscos da } \\
\text { vacinação }\end{array}$ \\
\hline 10 & $\begin{array}{l}\text { Nardi ACF et. } \\
\text { al }\end{array}$ & $\begin{array}{l}\text { Comunicação em } \\
\text { saúde: um estudo do } \\
\text { perfil e da estrutura das }\end{array}$ & $\begin{array}{l}\text { Investigar as } \\
\text { características dos } \\
\text { serviços de comunicação }\end{array}$ \\
\hline
\end{tabular}




\begin{tabular}{|l|l|l|}
\hline & $\begin{array}{l}\text { assessorias de em saúde, a partir da } \\
\text { comunicação } \\
\text { municipais em 2014- } \\
2015\end{array}$ & $\begin{array}{l}\text { Secretarias Municipais de } \\
\text { Saúde (COSEMS) no } \\
\text { Brasil }\end{array}$ \\
\hline
\end{tabular}

Fonte: Dados do estudo, 2019.

Quadro 2 - Categorias temáticas segundo as referências dos estudos incluídos. São Paulo - SP, Brasil. 2019.

Ao analisar os estudos, emergiram duas categorias conforme apresentado no Quadro 2.

\section{Categoria Temática}

1. Conscientização e incentivo à imunização

1. Comunicação Social em Saúde em prol da imunização

Fonte: Dados do estudo, 2019.

\section{CONSCIENTIZAÇÃO E INCENTIVO À IMUNIZAÇÃO}

Em estudos com abordagem qualitativos os indivíduos expressam diferentes significações sobre as normas brasileiras de imunização. Os pais que vacinam seus filhos revelam que imunizar os seus filhos é uma ação de responsabilidade. Pode-se observar o valor simbólico de responsabilidade parental, agregando a ideia de continuidade com o valor cultuado pelas tradições familiares e da sociedade. Sem minimizar o protagonismo da sociedade e a influência dos indivíduos no processo de imunização remetem uma ideia de que a sustentação e ênfase dado do ato de vacinar é a valoração cultural que está relacionada ao fato de ser obrigatório pela lei. ${ }^{(1,2)}$

Em estudos perspectivos, transversais e qualitativos apontam que cerca de $87,9 \%$ dos cartões de vacinação encontram-se atualizados, uma vez que as condições 
irregulares se dão por atrasos e perda do dia da vacina, argumentos esses considerados sem relevância. ${ }^{(3)}$

Visto que a conscientização e incentivo à imunização são extremamente relevantes e significantes, a Estratégia Saúde da Família está o nível da Atenção da Primária à Saúde, tendo a imunização como um de seus princípios e desejos como foco de trabalho. Ação essa de conscientização e incentivo dada pela atuação dos Agentes Comunitários de Saúde, que alertam as famílias e suas microáreas sobre a importância das vacinas. É considerado por tanto, com destaque, o espaço da Comunicação Social (Publicidade e Propaganda) veiculado ao Ministério da Saúde nos meios de comunicação de massa. Os agentes comunitários de saúde vêm desenvolvendo atividades de promoção da saúde e prevenção de doenças e agravos à saúde dos indivíduos, por meio de visitas domiciliares e de ações educativas individuais e coletivas nos domicílios e na comunidade, mantendo a equipe informada, principalmente a respeito daquelas em situação de risco. ${ }^{(3,6)}$

A Atenção Primária à Saúde está diretamente ligada às ações de Educação em Saúde, principalmente no que se diz respeito ao incentivo e conscientização da população em relação à imunização. A imunização deve ser entendida como um agente de modificação do processo de saúde-doença, pois garante a promoção e proteção à saúde e proteção da saúde dos indivíduos. A imunização tem sido a forma de prevenção de doenças. ${ }^{(5,6,7)}$

Em ações de conscientização e incentivo a imunização destaca-se o Enfermeiro em atuar diretamente em atividades e ações educativas com a população. O Enfermeiro é quem consegue ter maior vínculo com os usuários dos serviços de saúde, evidenciando a grande responsabilidade desse profissional no que se trata à imunização, ou seja, neste sentido o Enfermeiro e a equipe multiprofissional que atua em salas de vacina e ações educativas tem a autonomia de intervir no processo de saúde-doença de forma eficiente, contribuindo para que os usuários adotem o serviço de saúde e busquem sempre pela promoção e manutenção da saúde em todo o processo do cuidar no ciclo vital. ${ }^{(5.6,7)}$ 
Ocorrem falhas em que a imunização não ocorre por falta de aperfeiçoamento dos profissionais, gerando a diminuição no aperfeiçoamento profissional qualificação. E para garantir a imunização, os profissionais e usuários do serviço de saúde devem sempre estar informados quanto às indicações, contraindicações e efeitos da vacina, com o intuito de reduzir casos de atraso ou perda da vacina. Desta forma, devem-se reorganizar os serviços de saúde, proporcionando o planejamento das unidades. ${ }^{(5,6,7)}$

\section{COMUNICAÇÃO SOCIAL EM SAÚDE EM PROL DA IMUNIZAÇÃO}

A Comunicação Social em Saúde pode ser compreendida como a prática social que advém a interação dos seres humanos, por meio de falas, escritas sendo classificadas como verbal e não verbal. No Sistema Único de Saúde (SUS) nota-se uma dificuldade entre o preceptor e o emissor, dificuldades essas recorrentes a linguagens e saberes diferentes, que nem sempre são compartilhados. Esses fatores diferenciam-se por ordem sociocultural e estágios de desenvolvimentos cognitivos. ${ }^{(8)}$

Procura-se entender a inteligência, racionalidade e o desempenho do indivíduo. O princípio geral da inteligência é auxiliar a adaptação ao ambiente, podendo variar de acordo com o ambiente, hábito e reflexos. Ação comunicativa trata-se de um contexto dinâmico no qual podemos encaixar valores éticos e morais. A partir da relevância da Comunicação Social como um instrumento principal, os trabalhadores do Sistema Único de Saúde necessitam de suportes e implementações para a comunicação efetiva dialogada. ${ }^{(8)}$

A Comunicação Social proporciona um processo de compartilhamento e ajuda entre o profissional de saúde e o usuário, estabelecendo um processo de ajuda ao indivíduo e à família. Este processo de compartilhamento é caracterizado por atitudes de sensibilidade, aceitação e empatia entre os profissionais e usuários do serviço de saúde. A comunicação oferece condições significantes e relevantes para as boas práticas de promoção da saúde, tornando o estímulo do autocuidado e o cuidado dos usuários do serviço de saúde. ${ }^{(8)}$ 
A comunicação terapêutica é capaz de ajudar os indivíduos a enfrentar situações temporárias, ajustando-os à realidade, favorecendo o tratamento e o desenvolvimento dos usuários, tornando-os ativos no processo de cuidar. Porém, acredita-se que os aspectos de comunicação entre trabalhadores de saúde que integram as equipes, tanto no ambiente hospitalar como nos cenários de atenção primária, precisam ser investigados na procura dos problemas que permeiam as inter-relações dos profissionais com os usuários dos serviços de saúde. ${ }^{(8,9)}$

A Comunicação Social, em prol de campanha publicitária trás de forma impactante alerta para a importância de manter sempre a vacinação em dia. $O$ objetivo é mostrar que as baixas coberturas vacinais podem ser extremamente perigosas, já que abrem caminho para a reintrodução de doenças já eliminadas no país e que podem até aumentar as taxas de mortalidade. As campanhas publicitárias são consideradas impactantes, pois mostram casos reais de pessoas que sofrem até hoje pela não vacinação. $(8,10,11,12)$

No que se diz respeito a Comunicação Social ligada diretamente a Publicidade e Propaganda é possível expor alguns cenários onde pela primeira vez, a mascote das campanhas de vacinação do Ministério, o Zé Gotinha, aparece em um tom sério e preocupado. Disseminar informação para o grande público é sempre um desafio para a Saúde Pública. Por isso, a Saúde Brasil desenvolve campanhas educativas diferenciadas na área da saúde, cidadania e meio ambiente, com o objetivo de orientar a população divulgando informações para a promoção da saúde e qualidade de vida. As campanhas são de interesse social, desenvolvidas em uma linguagem simples e acessível e por promoverem temas relevantes à sociedade ganham destaque em toda mídia sendo reconhecidas como ferramenta de utilidade pública em saúde. ${ }^{(8,10,11,12)}$

\section{CONSIDERAÇÕES FINAIS}

Os resultados levantados neste estudo estão associados diretamente com a atuação direta da Atenção Primária à Saúde, principalmente no que se diz respeito a Estratégia Saúde da Família que aproxima a comunidade com os serviços em saúde, sendo capaz então de ofertar através de ações educativas campanhas publicitárias para o 
incentivo e conscientização à imunização, ressaltando a importância da vacina em todo o processo do ciclo vital.

\section{REFERÊNCIAS}

1. Barbieri CLA et al. A (não) vacinação infantil entre a cultura e a lei: os significados atribuídos por casais de camadas médias de São Paulo, Brasil. Cad. Saúde Pública 2017; 33(2):e00173315. Acesso em: 21 de Julho de 2019. Disponível em: http://www.scielo.br/pdf/csp/v33n2/1678-4464-csp-33-02e00173315.pdf

2. Silva FS et al. Incompletude vacinal infantil de vacinas novas e antigas e fatores associados: coorte de nascimento BRISA, São Luís, Maranhão, Nordeste do Brasil. Cad. Saúde Pública 2018; 34(3):e00041717. Acesso em: 21 de Julho de 2019. Disponível em: http://www.scielo.br/pdf/csp/v34n3/1678-4464-csp-3403-e00041717.pdf

3. Ramos CF, et al. Cumprimento do calendário de vacinação de crianças em uma unidade de saúde da família. Rev Pan-Amaz Saude 2010; 1(2):55-60. Acesso em: 21 de Julho de 2019. Disponível em: http://scielo.iec.gov.br/pdf/rpas/v1n2/v1n2a06.pdf

4. Mizuta AH et al. PERCEPÇÕES ACERCA DA IMPORTÂNCIA DAS VACINAS E DA RECUSA VACINAL NUMA ESCOLA DE MEDICINA. Rev Paul Pediatr. 2019;37(1):34-40. Acesso em: 21 de Julho de 2019. Disponível em: http://www.scielo.br/pdf/rpp/v37n1/0103-0582-rpp-2019-37-1-00008.pdf

5. Martins KM, Santos WL, Álvares ACM. A importância da imunização: revisão integrativa. Rev Inic Cient Ext. 2019; 2(2): 28-33. Acesso em: 21 de Julho de 2019.

em:

https://revistasfacesa.senaaires.com.br/index.php/iniciacao-

cientifica/article/view/153

6. Maia JÁ, Menezes FA, Santos PAM. PERCEPÇÃO DOS PAIS SOBRE A IMPORTÂNCIA DE ACOMPANHAMENTO DO CRESCIMENTO E DESENVOLVIMENTO INFANTIL. DêCiência em Foco. ISSN: 2526-5946. 2017; 1(2): 53-63. Acesso em: 21 de Julho de 2019. Disponível em: 
http://revistas.uninorteac.com.br/index.php/DeCienciaemFoco0/article/view/74/ 30

7. De Morais Júnior SLA et. al. A evolução histórica do calendário vacinal brasileiro infantil. REVISTA ENFERMAGEM ATUAL | 2018; 85. Acesso em: 21 de Julho de 2019.2 Disponível em: https://revistaenfermagematual.com.br/index.php/revista/article/view/247/146

8. Coriolano-Marinus MWL et al. Comunicação nas práticas em saúde: revisão integrativa da literatura. Saúde e Sociedade [online]. 2014, v. 23, pp. 13561369. Acesso em: 21 de Julho de 2019. Disponível em: https://doi.org/10.1590/S0104-12902014000400019.

9. Vasconcellos-Silva PR et al. A sociedade de risco midiatizada, o movimento antivacinação e o risco do autismo. Ciência \& Saúde Coletiva, 20(2):607-616, 2015. Acesso em: 21 de Julho de 2019. Disponível em: http://www.scielo.br/pdf/csc/v20n2/1413-8123-csc-20-02-0607.pdf

10. Nardi ACF et. al. Comunicação em saúde: um estudo do perfil e da estrutura das assessorias de comunicação municipais em 2014-2015* Epidemiol. Serv. Saude, Brasília, 27(2):e2017409, 2018. Acesso em: 21 de Julho de 2019. Disponível em: https://www.scielosp.org/article/ssm/content/raw/?resource_ssm_path=/media/ assets/ress/v27n2/2237-9622-ress-27-02-e2017409.pdf

11. Toscano C. Cartilha de vacinas: para quem quer mesmo saber das coisas / Cristina Toscano, Ligia Kosim . - Brasília: Organização Pan-Americana da Saúde, 2003. 40p. ISBN: 85-87943-29-4. Acesso em: 21 de Julho de 2019. Disponível em: http://bvsms.saude.gov.br/bvs/publicacoes/cart_vac.pdf

12. Brasil. Ministério da Saúde. Departamento de Vigilância das Doenças Transmissíveis. Manual de Normas e Procedimentos para Vacinação. Brasília : Ministério da Saúde, 2014. 176 : il. ISBN 978-85-334-2164-6. Acesso em: 21 de Julho de 2019.2 Disponível em: http://bvsms.saude.gov.br/bvs/publicacoes/manual_procedimentos_vacinacao .pdf

13. Mendes KDS, Silveira RCCP, Galvão CM. REVISÃO INTEGRATIVA: MÉTODO DE PESQUISA PARA A INCORPORAÇÃO DE EVIDÊNCIAS NA SAÚDE E NA 
ENFERMAGEM. Texto Contexto Enferm, Florianópolis, 2008 Out-Dez; 17(4): 758-64. Acesso em: 08 de Agosto de 2019. Disponível em: http://www.scielo.br/pdf/tce/v17n4/18.pdf

14. Câmara $\mathrm{RH}$. Análise de conteúdo: da teoria à prática em pesquisas sociais aplicadas às organizações. Gerais: Revista Interinstitucional de Psicologia, 6 (2), jul - dez, 2013,179-191. Acesso em: 08 de Agosto de 2019. Disponível em: http://pepsic.bvsalud.org/pdf/gerais/v6n2/v6n2a03.pdf

Enviado: Agosto, 2019.

Aprovado: Outubro, 2019. 